\title{
Intradimensional and extradimensional shift performance of children in a differential conditioning task ${ }^{\prime}$
}

HOWARD L. GARBER AND LEONARD E. ROSS UNIVERSITY OF WISCONSIN

The intradimensional and extradimensional shift performance of preschool children was compared with that of second graders in a differential conditioning (single cue presentation) situation. The younger children showed better ID than ED shift performance and their ID-ED difference was greater than that of the older children despite the fact that the differential conditioning paradigm prevents simultaneous comparison of dimensional cue values.

Early studies comparing reversal and nonreversal shift performance following discrimination learning were interpreted (e.g., Kendler \& Kendler, 1962) as indicating that the relative shift performance of children of different ages is related to a transition to verbally facilitated behavior during the 5-7 age range. Recently, however, several authors have questioned the reversal-nonreversal paradigm on methodological grounds and have presented data contrary to a mediational, verbal-transition prediction. With respect to methodology it has been suggested that intradimensional (ID) and extradimensional (ED) shift comparisons, rather than reversal-nonreversal comparisons, provide the proper test for the presence of mediational or attentional processes (see Shepp \& Turissi, 1966) with superior ID performance indicating the operation of these processes. Empirically, ID shifts have been found superior to ED shifts with rats (Shepp \& Eimas, 1964), and preschoolers (Dickerson, 1966). In addition Eimas (1966) found ID superiority with kindergarten children that was as great or greater than that with second graders.

While the methodological considerations and data cited raise serious questions about the developmentalmediational interpretation of discrimination shift performance, there are alternate possibilities that might account for the lack of human-infrahuman or human developmental differences in ID and ED shift performance. One possibility is that the two-choice simultaneous discrimination situation, because it permits the simultaneous comparison of both cues on a dimension, so facilitates verbal or attentional "dimensionalizing" that the ID-ED paradigm is insensitive to any verbal-mediational differences that occur with age. The purpose of the present study was to examine this possibility by comparing the ID and ED shift performance of preschoolers and second graders with a differential conditioning (singlecue presentation) paradigm, where only a portion of the cue set is available at one time and simultaneous comparison of cues on a dimension is impossible. In this situation the tendency to develop dimensionspecific mediational responses should be reduced and developmental differences in mediation might be expected to appear as differential ID-ED performance. Two levels of original learning (OL), criterion or criterion plus 25 trials of overtraining (OT), were included in the design and a response (lever lifting) was used which permitted its amplitude characteristics to be recorded.

Method

The $\mathrm{Ss}$ were 64 preschool children (mean $\mathrm{CA}=\mathbf{5 6 . 9}$ months; range, 44-67 months) and 64 second graders (mean $\mathrm{CA}=94.9$ months; range, 82-113 months) attending various nursery and elementary schools in Madison, Wisconsin.

The apparatus consisted of a large plywood box, with a handle which served as the response manipulandum. The handle was connected to a pivot so that it could be operated freely in a $21 \mathrm{~cm}$ vertical slot. When the handle was lifted by $S$ to a criterion $(7 \mathrm{~cm})$ height, a photocontact relay was activated and either a reward of M\&Ms for correct responses, or a buzzer for incorrect responses, was delivered. Movements of the handle produced a linear recording of response amplitude on a Texas Instruments polygraph. Directly beneath the handle was a single In-line readout display unit, which presented the color and form discriminanda. The stimuli used were four colors (blue, yellow, red, and green) and four forms (an $X$ within a square, a circle, a solid square, and a plus sign), the colors and forms being divided into two cue sets. Blue and yellow, and $\mathrm{X}$-squares and circles, were used as the OL stimuli. The other four stimuli composed a second cue set and were used in the shift to replace the cue set used in OL.

All Ss were first trained to a criterion of $9 / 10$ correct responses on the OL task. After attaining criterion in $O L$, each $S$ received one of four experimental conditions, according to the group to which he had been assigned. Two of these groups were immediately given the shift task, half receiving an ID, and half receiving an ED shift task. The remaining two groups (within each age group) received an additional 25 (OT) trials, after which half were presented with an ID and half with an ED shift. If the shift task was an ID shift, then the relevant dimension (form or color) was the same as OL; if the shift 
task was an ED shift, the relevant dimension (form or color) was the originally irrelevant dimension of OL. Relevant dimension and positive cue were balanced within each group. Cues of both dimensions were replaced in the shift for all Ss in order to control for novelty, partial reinforcement, and instrumental response transfer effects in the shift phase.

On each trial the discriminandum was a compound stimulus composed of one cue from the relevant dimension and one cue from the irrelevant dimension. The presentation of each cue on each trial was according to a Gellerman series, with each irrelevant cue paired with each relevant cue on $50 \%$ of the trials.

Subjects were told they were going to play a game in which they could win candy by lifting the handle only when the right light was on. If $S$ did not make a $7 \mathrm{~cm}$ response during the $5 \mathrm{sec}$ when the positive discriminandum was present, or if $S$ made such a response to the $5 \mathrm{sec}$ negative discriminandum, an error was recorded for that trial. If $\mathrm{S}$ did not attain the criterion of $9 / 10$ correct responses by the 80 th OL trial, the $\mathrm{S}$ was given special instructions and then allowed to attain criterion, whereupon OT or shift was immediately introduced. In the shift condition, the session was terminated if the $S$ did not reach the $9 / 10$ criterion in 80 trials.

\section{Results and Discussion}

An analysis of variance of errors made in original learning revealed that older children learned the $O L$ problem faster $(p<.001)$ than younger children. When color was the relevant dimension, the task was more difficult $(p<.001)$ than when the relevant dimension was form. The amplitude data was of quite limited value due to the all-or-none nature of most of the responses. The only amplitude data of note was the greater OL response amplitude found for fast learners (defined as Ss whose error score was lower than their OL group median) as compared to slow learners $(\mathrm{p}<.01)$.

The mean errors made by the various subgroups during the shift phase were as follows, with the criterion group mean presented first and OT group second: Preschool ID, 7.15, 8.35; Preschool ED 20.1, 19.05; Second Grade ID 6.45, 2.40; and second Grade ED $13.15,6.25$.

An analysis of variance of these data, including the factors of age, shift, degree of training, dimension, and sex, showed not only that the ID shift task was easier than the ED shift task overall $(p<.001)$, but also that the younger children had a greater ID superiority, i.e., a significant shift by age group interaction was found $(p<.05)$, with subsequent Newman-Keuls tests finding a significantly greater number of ED errors by young Ss as compared to the older group, and a significant ID-ED difference for the younger groups (all $\mathrm{p}$ values $<.05$ ). The ID-ED difference for older children approached but did not reach the .05 level.

Neither the degree of training main effect, nor interactions involving this factor, reached the .05 level, but the degree of training by age interaction did reach the .10 level. This interaction reflected the fact that OT had little effect on the younger Ss but facilitated both the ID and ED shift performance of the older children. The latter finding is not predicted from simple mediation theory but has been reported previously (e.g., Eimas, 1966).

It is clear from these data that the use of a differential conditioning paradigm, even with its lack of opportunity for simultaneous comparison of cues, did not produce the results predicted from developmentalmediation theory. As had previously been found with simultaneous discrimination situations, the ID shift superiority was as pronounced, or more so in this case, with young children who presumably were mediating less.

Several possibilities remain. For example, the ID superiority may reflect attentional processes which do not change developmentally in an important way, in terms of consequences for these paradigms, or a combination of attentional and verbal mediation factors may have been involved in which either was sufficient to produce the effect. Other processes may also compensate for a mediational deficit in the preschool children. Of possible relevance to this is the observation that the younger children verbalized OL solutions that involved the memorizing of compounds to a much greater extent than did the second graders. It is not clear, however, just how such compounding might produce or facilitate ID-ED differences.

\section{References}

DICKERSON, D. J. Performance of preschool children on three discrimination shifts. Psychon. Sci., 1966, 4, 417-418.

EIMAS, P. D. Effects of overtraining and age on intradimensional and extradimensional shifts in children. J. exp. Child Psychol, $1966,3,348-355$.

KENDLER, H. H., \& KENDLER, T. S. Vertical and horizontal processes in problem solving. Psychol. Rev., 1962, 69, 1-16.

SHEPP, B. E., \& EIMAS, P. D. Intradimensional and extradimensional shifts in the rat. J. comp. physiol. Psychol, 1964, 57, 357-361.

SHEPP, B. E., \& TURISSI, F. D. Learning and transfer of mediating responses in discrimination learning. In N. R. Ellis (Ed.), International review of research in mental retardation. Vol. 2 . New York: Academic Press, 1966. Pp. 85-121.

Note

1. Supported by PHS Grant MH 10235 and PHS Training Program HD 00117 . This study was based on a thesis submitted to the Graduate School of the University of Wisconsin by the first author in partial fulfillment of the requirements for the M. S. degree. The authors wish to express their appreciation for the cooperation of the Madison Neighborhood House, Tiny Tot Play School, and St. James Elementary School, Madison. 